\title{
LA SUBSUNCIÓN Y EL MODO DE CONSUMO CAPITALISTA
}

\author{
The Subsumption and the Capitalist Mode of Consumption
}

\author{
Xavi Cava \\ Seminari de Filosofia Política \\ Universitat de Barcelona \\ xavicava@gmail.com
}

\section{Resumen:}

El consumo fue uno de los grandes temas olvidados de la economía política y de su crítica por parte de Karl Marx. Tampoco la tradición marxista posterior se preocupó por rellenar ese hueco teórico de una forma clara. Solo a partir de la segunda mitad del siglo XX se han dado algunos pasos, más bien tímidos, en este sentido. Así las cosas, este artículo se postula precisamente como una aportación a la reflexión sobre el consumo en clave marxista. Dada la amplitud de este campo de investigación, vamos a centrarnos en una cuestión específica: discutiremos las características de lo que vamos a describir como el modo de consumo capitalista, los principales hitos de su desarrollo histórico, y cuáles han de ser sus condiciones de posibilidad.

\section{Palabras clave:}

Modo de consumo, subsunción, producción, reproducción, trabajo de consumo, capitalismo.

\begin{abstract}
:
Consumption was a topic largely forgotten on the classic political economy and its critic by Karl Marx. Later Marxist tradition has paid little attention to it, at least until the second half of the twentieth century. In this paper, I will contribute to the discussion about consumption from a Marxist point of view. The topic is too broad, so I will focus on a specific question: the main traits of what I will call the capitalist mode of consumption, its historical development, and its conditions of possibility.
\end{abstract}

\section{Keywords:}

Mode of consumption, Subsumption, Production, Reproduction, Work of consumption, Capitalism. 


\section{LAS FUNCIONES DEL CONSUMO PARA EL CAPITAL}

Tratemos por un instante de considerar al consumo estrictamente desde el punto de vista del capital: en esta perspectiva, lo que se nos aparece es que el consumo cumple dos funciones esenciales para ese capital. La primera función es la de dar el "remate final" a la valorización del capital. En la fase de adquisición de mercancías, bienes o servicios, el consumidor, actuando como comprador, transforma el capital que está materializado en la mercancía en capital líquido, en capital en forma de dinero. Durante la fase productiva, el papel activo en el proceso está en manos del capitalista, que es quien adquiere, organiza y pone a trabajar los diferentes elementos que intervienen en la producción. Pero al llegar a la fase de consumo, la iniciativa pasa al consumidor, quien decide qué y cuándo consumir.

Por otro lado, sabemos que el valor de la mercancía no se limita a la suma simple del valor de los elementos que intervienen en su producción (materias primas, instrumentos de producción, fuerza de trabajo). También forma parte suya el plusvalor. Esto implica que, cuando el consumidor adquiere la mercancía, al mismo tiempo actualiza la plusvalía, le da forma de dinero. Por decir así, la hace efectiva, la materializa monetariamente. Sin el acto de adquisición, la plusvalía se mantendría de forma potencial en la mercancía, no se haría efectiva, y por tanto se detendría el proceso de valorización del capital. De esta manera, se puede decir que la actividad del consumidor resulta, aunque sea de forma involuntaria para él, en la acumulación de capital.

La segunda función fundamental del consumo, por lo que respecta al capital, es la reproducción del conjunto de la fuerza de trabajo. En el aspecto temporal, esta función tiene lugar a continuación de la primera. Con "conjunto de la fuerza de trabajo" no nos vamos a referir, en este contexto específico, al conjunto de trabajadores, sean activos o no. Tampoco a la suma de esos trabajadores y de sus familias o de las personas dependientes económicamente de ellos. Nos vamos a referir a la totalidad de la fuerza de trabajo tal y como aparece para el capital. Esta totalidad se le aparece necesariamente bajo la forma de mercancías, enmarcada en un espacio simbólico al que se suele denominar mercado de trabajo.

Sabemos que fuerza de trabajo no es lo mismo que actividad de trabajo ni que trabajo realizado. La primera existe como capacidad, como un conjunto de habilidades y disposiciones disponibles para ser empleadas. El trabajo, en cambio, es la materialización de esas disposiciones. Esta materialización puede entenderse tanto en el sentido del proceso de realizarse o del pasar a acto de algo que existía potencialmente, como en el de la objetivación en un producto o servicio concretos. Ahora bien, para que la fuerza de trabajo cobre forma, para que se configure en los cuerpos de los trabajadores y trabajadoras, ha de pasar por un proceso que, en cierta forma, puede considerarse como "creador": el proceso o actividad de consumo en su dimensión productiva. $\mathrm{Y}$, a su vez, este proceso creador ha de culminar en un resultado final, es decir, la propia fuerza de trabajo en cuanto mercancía disponible para su adquisición por el capital.

En definitiva, al decir que lo que se reproduce mediante el consumo, para el capital, es el conjunto de la fuerza de trabajo, lo que pretendemos es destacar dos 
cosas. Primero, que lo que se está reproduciendo es potencia, una fuerza o capacidad virtual todavía sin realizar. Esta reproducción tiene lugar en el cuerpo de los trabajadores y trabajadoras, que es a la vez tanto dispositivo reproductor/reproducido como soporte material de la fuerza de trabajo. Segundo, que la forma más adecuada para comprender esa reproducción pasa por considerarla como un conjunto, como una totalidad que engloba la reserva de energía y creatividad humanas que el capital a) necesita o podría llegar a necesitar, y que b) debe ser repuesta en un proceso incesante. Por supuesto, el consumo es mucho más que la simple reproducción de la fuerza de trabajo. Y, por supuesto, la reproducción de la fuerza de trabajo puede considerarse desde otros muchos puntos de vista. Pero insistimos: considerado desde el lado del capital, lo esencial respecto del consumo es que reproduzca el conjunto de la fuerza de trabajo y que transforme en capital líquido el plusvalor.

Por otro lado, el consumo en el capitalismo aparece estructurado en base a una triada fundamental: salario, mercancía y mercado. El salario supone, como es evidente, la existencia del trabajo asalariado. Una fuerza de trabajo libre obligada, como condición para su existencia, a ofrecerse al mercado es una condición fundamental para que exista el consumo en su forma capitalista. Forma parte de la naturaleza de esa fuerza de trabajo el que solo pueda reproducirse mediante mercancías; el que para disponer de los medios para conseguir esas mercancías, deba venderse a sí misma como mercancía; en fin, el que tenga la obligación ineludible de acudir al mercado tanto para obtener las mercancías como para ofrecerse a sí misma como una mercancía más.

Se podrá argumentar que, en las sociedades capitalistas postfordistas, existen muchas otras formas de obtener ingresos destinados al consumo: subsidios, pensiones, créditos, y demás. Pero, en última instancia, todos ellos acaban por depender de la riqueza creada gracias al trabajo, sea de forma directa o indirecta. Se solía afirmar que el trabajador (o el proletario, si se prefiere) es aquel que no tiene nada más que su fuerza de trabajo para vender. Si invertimos esta afirmación, se puede decir que el trabajador es aquel que está obligado a vender su fuerza de trabajo para poder acceder a sus medios de consumo. Porque su desposesión es doble, recordemos: está separado de los medios de producción, pero también lo está de los medios de consumo.

Los medios de consumo, en el capitalismo, son producidos en la forma de mercancías. Entre otras cosas, esto significa que no son bienes o servicios producidos para ser consumidos de forma inmediata. Son producidos para, en primer lugar, ser vendidos, y sólo a continuación ser consumidos. Considerado desde la perspectiva del consumidor, esto significa que la forma de la mercancía se instituye como una mediación que se interpone entre él y el bien de consumo. Esta mediación disuelve la inmediatez entre la producción y el consumo que caracterizaba a la mayoría de modos de producción pre capitalistas o, simplemente, no capitalistas. El pequeño agricultor libre ateniense o romano, el artesano de las ciudades antiguas y medievales, disponían inmediatamente del fruto de su trabajo (descontados impuestos, diezmos y otras servidumbres). Podían consumirlo directamente, o bien intercambiarlo por otros productos. La forma mercancía, por el 
contrario, imprime la etiqueta de "propiedad ajena" sobre el conjunto de lo producido en el capitalismo. Forma social por excelencia, la mercancía abre primero una separación entre bienes producidos y trabajadores, para reunirlos después en la institución social constituida como el espacio simbólico en el que se desarrolla su intermediación: el mercado.

En el capitalismo, el mercado es a la vez un espacio formal/abstracto y material/concreto. En tanto que espacio abstracto, es el lugar en el que se ponen en conexión los valores de las mercancías y se los hace circular. La función que cumple en este sentido es la de actuar como una institución económica y social. En tanto espacio material, es el lugar en el que esa función se hace concreta, donde los valores abstractos de las mercancías adquieren corporeidad y se hacen visibles. Por lo que hace al consumo, la conexión entre los valores de las mercancías se organiza en torno a dos grandes esferas interrelacionadas: la que corresponde a la mercancía "fuerza de trabajo", y la que corresponde a las mercancías producidas mediante esa fuerza de trabajo (es decir, el resto de mercancías). El mercado aparece, de esta forma, como un espacio de abstracción para el trabajador/consumidor: un espacio en el que se abstrae a sí mismo de su dimensión de trabajador o trabajadora, de humanidad portadora de fuerza de trabajo, y en el que abstrae a las mercancías de su naturaleza de bienes producidos mediante la fuerza de trabajo.

Hagamos notar, finalmente, que los tres elementos constituyentes del consumo (salario, mercancía y mercado) se relacionan gracias a un mecanismo mediador que los atraviesa por igual: el dinero. Los salarios, como los valores de las mercancías, se expresan en precios y se materializan en dinero. El mercado, por último, es el espacio "natural" del dinero, el lugar en el que circula poniendo en conexión todos los elementos.

\section{LA CARACTERIZACIÓN HISTÓRICA DEL MODO DE CONSUMO CAPITALISTA}

La triada salario-mercancía-mercado es condición necesaria, pero no suficiente, para que exista algo tal que un modo de consumo capitalista. Estas tres categorías deben, además, aparecer organizadas de manera tal que puedan Ilevar a cabo las dos funciones que hemos señalado más arriba: valorizar el capital y reproducir la fuerza de trabajo. La necesidad de esta estructura se nos aparecerá más claramente si volvemos la vista al pasado y examinamos épocas históricas en las que salarios, mercancías y mercados aparecieron simultáneamente, e incluso llegaron a instituirse en una cierta estructura, pero no alcanzaron a madurar en un modo de consumo similar al capitalista.

Vamos a considerar un ejemplo histórico: la Atenas de los siglos V y IV a.C, la Atenas de Pericles y Platón. Empecemos señalando que, en esta época, el ágora ateniense experimentó una notable transformación en su función económica y social que nos va a proporcionar muchas pistas sobre la forma de consumo que se llegó a constituir allí. Recordemos que, en las ciudades griegas, el ágora era tradicionalmente el centro vital de la sociedad, el núcleo administrativo, político y económico. Era el lugar en el que los varones griegos, además de discutir acerca de lo terrenal y de lo divino, hacían tratos y se dedicaban a la política. Pero también 
era el lugar donde las mujeres y los esclavos eran enviados a intercambiar los excedentes agrícolas; y donde los metecos (los ciudadanos extranjeros con permiso de residencia) podían vender su producción artesanal. En la mayoría de ocasiones, la base formal de estos movimientos económicos era el trueque, el intercambio de una cierta cantidad de un producto a cambio de una cantidad de otro producto según proporciones más o menos fijas. Este fue el mecanismo dominante hasta que, hacia mediados del siglo VI a.C Ilegó a Grecia un invento que iba a revolucionar este sistema de intercambio: el dinero acuñado en la forma de monedas.

El uso de la moneda acuñada tuvo un éxito fulgurante, y para finales del siglo VI a.C su difusión se había extendido por la práctica totalidad del mundo griego. Ahora bien, en Atenas el dinero acuñado acabó teniendo un papel económico mucho más intenso y complejo que en el resto de poleis griegas. A grandes rasgos, esto fue consecuencia de la prosperidad que trajo el imperio naval y comercial sobre el que se asentó la democracia ateniense en el siglo $\mathrm{V}$ a.C. Pero hubo dos razones especialmente destacadas que explican el particular progreso de la función de la moneda en Atenas. En primer lugar, el principal puerto ateniense, el Pireo, se fue convirtiendo durante el siglo $\mathrm{V}$ a.C en el centro más importante del tráfico naval mercantil en el Egeo oriental. Como consecuencia de esta intensificación del comercio, la circulación de monedas en la ciudad no solo se multiplicó, sino que en cierta forma se "democratizó": el dinero dejó de ser un instrumento monopolizado por las élites políticas y militares, y empezó a ser un instrumento cotidiano para buena parte de la población de la ciudad. En segundo lugar, a partir de cierto momento el servicio militar en la marina de guerra ateniense empezó a ser remunerado monetariamente. Este servicio solía durar ocho meses al año, y se cree que en él pudieron llegar a participar, en épocas de guerra, un mínimo de 20.000 hombres, entre remeros y marineros (inicialmente todos ciudadanos libres, aunque según el momento y la situación bélica llegó a ser necesario reclutar a metecos e incluso esclavos). Pues bien, el estado ateniense acabó por pagar un salario fijo a cada ciudadano movilizado durante este servicio militar, pago que era considerado como una compensación por el tiempo que los ciudadanos no podían dedicar a mantener su hogar, su oikos.

Encontramos, entonces, que un porcentaje considerable de la población masculina adulta ateniense recibió lo que se podría considerar un salario estacional, pero constante, a lo largo de bastantes años. Un salario en forma monetaria, y cuyo destino debía ser, en una proporción apreciable, la adquisición de medios de consumo para el ciudadano y su familia. Estos medios de consumo sustituían a aquellos otros que, en otras circunstancias, hubiese podido producir el propio ciudadano con la ayuda de su familia. Destaquemos que, hasta donde alcanza nuestro conocimiento, esta situación representaba una novedad histórica, pues hasta entonces en el mundo griego el pago por la prestación de un servicio militar había sido algo esporádico y generalmente restringido a la contratación de mercenarios bárbaros.

Tenemos, al mismo tiempo, una ciudad próspera y en expansión, a la que afluían mercancías de todos los rincones del Mediterráneo. Estas mercancías tenían un precio que ya era establecido en base al dinero acuñado como unidad de medida, 
algo que también representaba una novedad. Y tenemos, finalmente, un ágora que, bajo la más que probable influencia de estos factores (difusión del dinero, salarios constantes y una cierta circulación de mercancías) evolucionó hacia un sistema de mercado institucionalizado, en el que los intercambios ya no se basaban solo en el trueque, sino que cada vez más lo hacían en función de un sistema de precios estables. Por tanto, en el ágora ateniense se instituyó un mercado en el doble sentido que hemos mencionado: ya no solo como el lugar físico, concreto, donde se realizaban los intercambios, sino también como una institución económica, como un espacio abstracto en el que se ponían en conexión y circulaban los valores de las mercancías. Un espacio organizado según unos principios y bajo unas determinaciones diferentes a las del antiguo intercambio simple.

¿Es posible, a partir de todo esto, afirmar que en Atenas germinó un modo de consumo equivalente, o como mínimo muy similar al capitalista? Por supuesto que no. A pesar de las similitudes, incluso a pesar de las tendencias que parecían desarrollarse en ese sentido, existían al menos dos grandes obstáculos que impidieron el desarrollo de un modo de consumo capitalista o "proto-capitalista". El primero fue la práctica inexistencia del trabajo asalariado. El servicio en la marina estaba remunerado, cierto, pero no era un trabajo productivo. La producción agrícola estaba mayoritariamente en manos de los pequeños propietarios libres y sus familias, ayudados a lo sumo por el trabajo de algún esclavo. Lo mismo sucedía con la mayor parte de la producción artesanal, con la salvedad de que generalmente esta esfera de la producción se reservaba a los metecos.

Por otro lado, una parte sustancial del trabajo más pesado, tanto en Atenas como en el resto de Grecia, era realizado por la mano de obra esclava, que llevaba a cabo así la función que, de haber existido en una cantidad adecuada, le hubiese correspondido realizar a la mano de obra asalariada. La figura del trabajador libre, pero sin propiedades, fue siempre minoritaria en la Grecia clásica. Y eso que las condiciones para el surgimiento de una clase agrícola asalariada se dieron: de hecho, la política ateniense de pagar un salario a los ciudadanos que servían en la flota fue, en gran medida, una forma de aliviar las tensiones sociales que provocaba la tendencia a la concentración de la tierra en manos de cada vez menos propietarios. $\mathrm{Y}$ es que los campesinos desposeídos de sus tierras preferían convertirse en remeros y arriesgar su vida en la guerra, a trabajar en las tierras de otros. La razón es que el trabajo por cuenta ajena era considerado una ocupación indigna de un hombre libre, además de ser míseramente remunerada (a medio plazo era más rentable comprar un esclavo que pagar un sueldo fijo a un trabajador). En definitiva, la institución de la esclavitud fue un obstáculo insalvable para el desarrollo de una mano de obra libre asalariada, y por tanto, para un modo de consumo estructurado sobre la reproducción de ese tipo de mano de obra.

El segundo obstáculo fue la naturaleza estática de la economía antigua. Esta naturaleza se refleja en el ideal de auto-suficiencia que dominó el imaginario colectivo griego. En una economía fundamentada en la agricultura, la producción de la mayoría de propiedades alcanzaba justo para cubrir las necesidades de una familia; no se producía para el mercado, mucho menos para la exportación a mercados lejanos. Cierto que hubo excepciones puntuales, pero nunca pasaron de 
tales. Tampoco la tendencia histórica a la concentración de propiedades agrícolas en manos de la aristocracia, aunque resultó en una tendencia a la pauperización de la figura del pequeño propietario, cambió las cosas. Las crisis sociales recurrentes que esta tendencia generaba no evolucionaron hacia la formación de un proletariado rural o emigrado a la polis. Por lo general, el problema tendió a ser resuelto mediante mecanismos expeditivos como las migraciones masivas, y a menudo forzadas, hacia colonias o, en el caso de algunas democracias, mediante una cierta política de redistribución social de la riqueza (como fue la remuneración del servicio en la flota en el caso específico de Atenas).

En definitiva, la inmensa mayoría de la población en Grecia trabajaba para producir justo lo que necesitaba, y a la inversa, necesitaba consumir poco más de lo que producía. La idea de incrementar la producción más allá de lo estrictamente necesario con el objetivo de conseguir un aumento constante de la riqueza fue totalmente ajena a la organización de la economía antigua y, por tanto, ajena a la lógica que gobernaba las acciones de los antiguos. En otras palabras, ni hubo ni pudo haber producción de plusvalor, como tampoco la necesidad de un incremento constante del consumo para sostener el ritmo de crecimiento de un supuesto capital. Esto no significa que no existiese una explotación económica, particularmente de la fuerza de trabajo esclava. Pero esta explotación implicó un modo de consumo muy diferente al capitalista.

\section{EL PROCESO HISTÓRICO DE DESARROLLO DEL MODO DE CONSUMO CAPITALISTA}

Insistamos en ello: no basta con que coincidan salarios, mercancías y un mercado institucionalizado para poder hablar de un modo de consumo capitalista. Es necesario que, además, estos elementos se organicen bajo una determinada formación socioeconómica. Una formación estructurada, precisamente, en base al modo de producción del capitalismo industrial. Solo subsumido a ese modo de producción puede el consumo realizar adecuadamente las funciones de valorización del capital y reproducción de la fuerza de trabajo. Como vamos a ver a continuación, el proceso de subsunción del consumo, que se consuma en su configuración final como modo de consumo capitalista, corre paralelo al desarrollo del mismo capitalismo, y experimenta su impulso decisivo durante la segunda etapa de la industrialización capitalista.

Aunque las dos funciones del consumo son necesarias por igual para el desarrollo del capital, la clase burguesa tardó mucho más tiempo en tomar conciencia teórica de la función reproductora que de la función valorizadora. La razón principal de esta diferencia, por supuesto, es que en su primera etapa el capitalismo industrial apenas estaba iniciando su expansión, y sus necesidades de mano de obra eran todavía limitadas. El problema de la reproducción de la clase trabajadora era una cuestión aparentemente irrelevante y que, en cualquier caso, podía dejarse en manos de los "instintos naturales" de los propios trabajadores y trabajadoras. Pero a esta despreocupación también contribuyó el hecho de que la fuerza de trabajo se caracteriza por ser una mercancía con una especificidad doble, especificidad que la diferencia del resto de mercancías. Por un lado, se caracteriza por esa bien conocida capacidad de añadir más valor a las mercancías que produce del que cuesta su

OXÍMORA REVISTA INTERNACIONAL DE ÉTICA Y POLÍTICA 
propia reproducción como mercancía (en forma de salario). Pero, por otro lado, la fuerza de trabajo también se caracteriza por su capacidad para reproducirse a sí misma, por ser su propio agente productivo. Una capacidad, digamos, auto-poiética, que el trabajador lleva a cabo mediante la actividad de consumo. Gracias a esta capacidad auto-productiva, en los inicios del capitalismo industrial la reproducción de la fuerza de trabajo pudo dejarse a la iniciativa del propio trabajador. La mayoría de capitalistas ni consideraron la necesidad de ofrecer estímulos al consumo, y los únicos esfuerzos relevantes al respecto fueron las campañas de las damas de la alta burguesía contra el alcoholismo y el resto de vicios que, supuestamente, caracterizaban a la clase trabajadora.

Así pues, la capacidad "natural" de la familia trabajadora de reproducirse por sus propias manos fue paradójicamente determinante en que, en las etapas iniciales del capitalismo industrial, la burguesía no tuviese en cuenta la importancia del consumo de la clase trabajadora para la gestión del capital. No sólo tendió a menospreciar la forma y las condiciones en las que tenía lugar el consumo proletario, sino que, en su avidez por aumentar la tasa de plusvalía absoluta (aumentar la jornada laboral y reducir los salarios), incluso lo obstaculizó. Sin embargo, la propensión inicial a limitar la capacidad de reproducción de la clase trabajadora al mínimo imprescindible para su supervivencia hubo de ser modificada a medida que el capitalismo se desarrollaba. A partir de la expansión de la gran industria y la extensión del capitalismo por buena parte de Europa y Estados Unidos, en la segunda mitad del siglo XIX, la demanda de una mano de obra cada vez más cualificada y abundante fue una señal clara de que el consumo había de servir para algo más que para dar salida a la creciente producción.

Pero fue con la re-ordenación fordista de las relaciones productivas, a principios del siglo XX, cuando la tendencia se invirtió por fin. En esta etapa el consumo pasó a ser subsumido formalmente dentro del sistema productivo capitalista. Ante los progresos del sistema fordista, el simple incremento compulsivo de la producción con vistas a realizar la máxima cantidad "bruta", por así decir, de plusvalor se reveló como un modo de explotación obsoleto e ineficaz. El proceso de acumulación de capital debía racionalizarse, someterse a los mismos criterios de eficiencia y maximización de los recursos que estaban organizando la cadena de producción. Y el consumo debía jugar un papel fundamental en esta racionalización.

En este sentido, uno de los objetivos de la racionalización fordista consistió en incentivar el consumo de una clase trabajadora en la que se debía desarrollar una fuerza de trabajo cada vez más productiva y especializada. La subsunción formal del consumo pasaba por el incremento de salarios, la multiplicación de estímulos al consumo, y la generación de una auténtica ideología consumista alrededor. Paralelamente, el incremento sostenido de la producción lanzaba al mercado una cantidad cada vez más ingente de mercancías que exigían ser adquiridas: la función valorizadora del consumo no quedaba eclipsada por la función reproductora, sino que ambas eran conducidas a converger de forma cada vez más sincronizada. Por otro lado, la estrategia de racionalización también exigía formar lo que podríamos denominar una auténtica fuerza de consumo, es decir, una masa de individuos adiestrados para absorber la creciente oferta de mercancías. En esta época se forjó el 
concepto de "consumidor" de la mano de la publicidad y el marketing, se multiplicaron los nuevos espacios urbanos destinados a la venta, o se implementaron los primeros estudios estadísticos de comportamiento del consumidor.

Es necesario insistir en esta idea. A partir de un cierto momento, el trabajador necesitó ser formado ya no solo como trabajador, sino también como consumidor. En otras palabras, su reproducción como fuerza de trabajo ya no se podía dejar a su libre albedrío, a sus instintos naturales, o a una cultura heredada de unas raíces no urbanas. No fue casual que la necesidad de formar al consumidor coincidiese con la extensión de la educación universal, de la salud pública, de los primeros esbozos de un estado del bienestar, como también de la evolución de la publicidad de ser una actividad prácticamente artesanal, a convertirse en una industria cada vez más desarrollada. Podría llegar a decirse que, en esa fase del capitalismo, el modo de consumo capitalista se desarrolló para transformarse en una particular institución disciplinaria.

\section{EL POSTFORDISMO Y LA SUBSUNCIÓN TOTAL DEL CONSUMO}

La estructuración y la disciplinación de una fuerza de consumo, desarrolladas a partir del fordismo, tenían como objetivo último intensificar las dos funciones del modo de consumo capitalista, la valorización y la reproducción de la fuerza de trabajo. Puesto el proceso de valorización ha sido mucho más tratado por la tradición marxista, para finalizar vamos a centrarnos fundamentalmente en la manera en la que el capitalismo postfordista ha transformado de nuevo el proceso de reproducción de la fuerza de trabajo.

Decíamos antes que el trabajador o la trabajadora, como ser humano, dispone de esa maravillosa capacidad (maravillosa para el capital, se entiende) para reproducirse mediante su propio trabajo. Puesto que esa reproducción se realiza, fundamentalmente, a través del consumo de mercancías, al capital contemporáneo le interesa en primer lugar que el trabajador sea, sobre todo, un consumidor tan ávido como experimentado. En la figura del consumidor postfordista, sometido a los requerimientos del capitalismo cognitivo, el grado de refinamiento en el consumir es al mismo tiempo un signo claro de su aptitud como mano de obra. Un consumidor curioso, abierto a la novedad y creativo, es simultáneamente un trabajador curioso, abierto a la novedad y creativo.

Los mecanismos y estrategias diseñados para incentivar, dirigir y controlar la actividad de consumo son numerosos y muy efectivos; no los vamos a repasar ahora, como tampoco las múltiples formas de resistencia que se desarrollan como reacción. Nos interesa señalar, más bien, una aparente paradoja. Y es que, más allá de todas esas fuerzas invertidas en modular y modelar el consumo, el consumidor ha de mantener en todo momento una cierta autonomía en su actividad, un margen de libertad para que lleve a cabo el consumo por propia iniciativa. Esta autonomía resulta esencial para el modo de consumo del capitalismo postfordista. Veamos cómo.

Mediante su actividad el consumidor "asimila" las mercancías, las integra en sí. Esta asimilación ha de entenderse de una forma amplia: tiene lugar tanto en el 
ámbito fisiológico y emocional como en el ideológico y cultural. La importancia y la complejidad de este proceso se muestran de forma más evidente en el plano cultural, como ha puesto de relieve la tradición de estudios culturales en las últimas décadas. Pero tanto en un ámbito como en el otro estamos ante un mismo proceso de subjetivación mediada por las mercancías, un proceso que es característico de toda modo de consumo.

Proceso de subjetivación, de integración, o de interiorización si se prefiere; en cualquier caso su importancia para nosotros radica en que se lleva a cabo mediante una actividad que solo puede realizar el propio consumidor. Lo característico del modo de consumo capitalista es que en esta actividad, como decimos, debe haber tanto un grado de libertad y autonomía, como un grado de productividad. Solo mediante la combinación de ambos es posible que se realice el milagro cotidiano de la reproducción de la fuerza de trabajo asalariada. Milagro que consiste en que, a través de un consumo delimitado cuantitativamente por la capacidad de adquirir mercancías que establece el salario, el consumidor pueda reproducirse cualitativamente como una fuerza de trabajo cuyo valor real es superior a ese salario. $\mathrm{Si}$ se nos permite la ironía, podemos denominarlo el milagro de la reproducción ampliada de la fuerza de trabajo en el modo de consumo capitalista.

En el postfordismo la preservación de esta autonomía, característica de la actividad de reproducción consumidora, ha llegado a ser un elemento fundamental en la configuración del modo de consumo capitalista. El consumo postfordista está enredado en una contradicción insoluble entre la disciplinación capitalista y la autonomía productiva del consumidor. Un ejemplo de esta contradicción se manifiesta en uno de los mecanismos en los que se articula contemporáneamente la relación entre consumidor y mercancía. Ya en el fordismo se hizo evidente una tendencia a incrementar lo que podríamos denominar la "consumibilidad" de las mercancías. Con este concepto queremos referirnos al grado de disponibilidad, de aptitud para ser consumidas, asimiladas, utilizadas, con el que las mercancías son producidas y entregadas al mercado. Pensemos, como ejemplo, en la expansión de los platos de comida preparada: implican un mayor grado de consumibilidad frente a los alimentos sin preparar.

En el sistema productivo postfordista el incremento sostenido de la consumibilidad aparece como una tendencia aún más intensa. Ahora bien, a pesar de ello, incluso aquí la consumibilidad ha de tener un límite. El modo de consumo capitalista no puede (ni debe) sustituir por completo el gasto de energía física y/o psíquica que el consumidor debe realizar en el acto de consumo. Este esfuerzo o gasto de energía se caracteriza por una dimensión productiva, creadora, en el sentido de que sobrepasa la simple reposición de algo que existía previamente. Porque, al consumir, el consumidor no solo se está reproduciendo (recreándose a sí mismo como el ser que ya existe), sino que también se está produciendo (creando en sí y a partir de sí lo que todavía no forma parte de su ser). Esto nos lleva a postular, en definitiva, que la actividad consumidora ha de ser concebida como una forma peculiar de producción creadora: una producción que tiene lugar mediante lo que queremos denominar como trabajo de consumo. 
El trabajo de consumo ha sido siempre un elemento fundamental en la valorización del capital. En primer lugar, porque era el trabajo mediante el que se reproduce la fuerza de trabajo, mercancía necesaria para el proceso de producción. En segundo lugar, porque este trabajo de reproducción no le suponía al capital ningún coste añadido: era una actividad productiva que lleva a cabo el consumidor por su propia cuenta. Ahora bien, a partir de un cierto momento del desarrollo histórico capitalista fue necesario empezar a racionalizarlo, a optimizarlo. Hemos visto a grandes rasgos cómo ese trabajo de consumo llegó a ser subsumido formalmente por el capital. Llegados aquí, es necesario introducir un matiz respecto a ese proceso de subsunción. Porque el trabajo de consumo implica, en su origen, dos procesos paralelos derivados de una misma actividad. Por un lado, se encuentra la producción del ser humano en general. Por el otro, la reproducción de la fuerza de trabajo, de la capacidad productiva existente en potencia en el ser humano. Ambos, insistimos, arrancan de la misma actividad consumidora, pero pueden ser diferenciados analíticamente como dos cosas distintas: producción y reproducción.

Aproximadamente hasta la última etapa del fordismo, la reproducción de la fuerza de trabajo se mantuvo como una dimensión contenida en, y hasta cierto punto diferenciable, de la producción del ser humano. Durante el postfordismo, sin embargo, se fue difuminando la nítida distinción entre el ser humano como un todo, y aquella dimensión del ser humano que podría catalogarse como su fuerza de trabajo. Ha sido característico del postfordismo (o del capitalismo cognitivo, si se prefiere considerar así) la tendencia a que la totalidad de la vida, de la sociabilidad y la capacidad cognitiva humanas, pasen a formar parte de la reserva de fuerza de trabajo de la que el capital dispone o ha de poder disponer. De esta manera, en el consumo postfordista la simple reproducción (re-creación) de fuerza de trabajo acaba por expandirse hasta pasar a ser producción (creación) de una fuerza de trabajo continuamente mejorada, intensificada: "ampliada". Ahora bien, esta forma de subsunción tiene, como consecuencia inevitable, que se produzca el proceso opuesto: es decir, que la producción del ser humano tienda a reducirse, a su vez, a mera reproducción.

De esta manera, la especificidad del modo de consumo postfordista resulta determinada por la forma en la que se interrelacionan producción y reproducción, tanto de la fuerza de trabajo como del ser humano que es su fundamento. En términos generales, esta interrelación se caracteriza por una subsunción total del consumo que trasciende a la subsunción formal que tuvo lugar durante el fordismo. En función de esta subsunción total, el consumo contemporáneo ha de ser capaz de activar, de movilizar el conjunto de las capacidades humanas, y en particular, aptitudes como la creatividad, la sociabilidad o la comunicabilidad. En el modo de consumo que así se configura, el consumidor tiende a producirse a sí mismo bajo los rasgos específicos que definen la fuerza de trabajo característica del capitalismo postfordista: competencia comunicativa, auto-referencialidad productiva, empatía cognitiva, capacidad adaptativa, maleabilidad, predisposición al cambio y la novedad, etc.

Considerado desde la perspectiva del consumo, resulta irrelevante que una parte de la fuerza de trabajo así configurada no llegue a ser integrada en el sistema 
productivo. Esta es la paradoja final de la subsunción total del consumo: de la misma manera que, del lado de la producción, sigue siendo tan necesaria como inevitable la existencia de un ejército industrial de reserva, del lado del consumo ese ejército debe mantener una cierta capacidad de consumir; es decir, debe mantenerse como fuerza de consumo. Debe, por tanto, articularse como un "ejército consumidor de reserva". El consumidor despojado de un salario "digno" ha de conservar, a pesar de ello, una intensidad tal en su consumo que le permita reproducir e incluso producir ampliadamente sus cualidades como mano de obra postfordista. La disciplina de consumo le arrastra a suplir la limitación en el acceso a las mercancías (causada por la disminución de su "capacidad adquisitiva") con una intensificación de la productividad y la creatividad durante la actividad consumidora, es decir, del trabajo de consumo. Esto pasa, por ejemplo, por sustituir el consumo de mercancías de mayor consumibilidad (generalmente más costosas, pero que requieren menos trabajo de consumo) por el consumo de mercancías de menor consumibilidad (más baratas, pero cuyo "plus" de consumibilidad ha de surgir del propio trabajo). Surge como resultado la figura de un consumidor precarizado, reverso del trabajador precario, en la que se manifiesta una nueva dimensión de la pauperización que sigue generando estructuralmente el capitalismo. Esta figura informa ese ejército consumidor de reserva que puebla buena parte de las ciudades postmodernas, y que es el reflejo, desde el lado del consumo, de los efectos de la división internacional del trabajo imperante en el postfordismo.

\section{BIBLIOGRAFÍA}

ARISTÓTELES (2000). Política. Madrid, Gredos.

BAECK, Louis (1994). The Mediterranean Tradition in Economic Thought. London, Routledge.

BAUMAN, Zigmunt (2000). Trabajo, consumo y nuevos pobres. Madrid, Gedisa.

BOURDIEU, Pierre (2012). La distinción. Criterio y bases sociales del gusto. Madrid, Taurus.

FOUCAULT, Michel (2009). Nacimiento de la biopolítica. Madrid, Akal.

JENOFONTE (2000). Helénicas. Madrid, Gredos.

FINLEY, Moses (1973). La economía de la antigüedad. México, FCE.

KINLEY, David (1924) Money. A Study of the Theory of the Medium of Exchange. London, MacMillan.

LEBOWITZ, Michael A. (2005). Más allá de El capital. La economía política de la clase obrera en Marx. Madrid, Akal.

MARX, Karl (1976). El capital: crítica de la economía política. Grijalbo, Barcelona.

MARX, Karl (1971). Elementos fundamentales para la crítica de la economía política. (Grundrisse). Buenos Aires, Siglo XXI.

MEILLASSOUX, Claude (1975). Mujeres, graneros y capitales. México, Siglo XXI. 
MOSSÉ, Claude (1980). El trabajo en Grecia y Roma. Madrid, Akal.

MOSSÉ, Claude; VIDAL-NAQUET, Pierre; FERNÁNDEZ UBIÑA, José; GONZÁLEZROMÁN, Cristóbal (1977). Clases y luchas de clases en la Grecia Antigua. Madrid, Akal.

MORRIS, Ian (2005). The growth of Greek cities in the first millennium B.C. Recuperado de http://www.princeton.edu/ pswpc/papers/authorMZ/morris/morris.html.

NEGRI, Toni (2001). Marx más allá de Marx. Cuaderno de trabajo sobre los Grundrisse. Madrid, Akal.

PLATON (2000). República, Madrid, Gredos.

POLANYI, Karl; ARENSBERG, Conrad; PEARSON, Harry (1957). Trade and Market in the Early Empires. Chicago, The Free Press.

PRETECEILLE, Edmond y TERRAIL, Jean Pierre (1977). Necesidades y consumo en el capitalismo actual. México, Grijalbo.

RITZER, George (1996). La McDonaldización de la sociedad. Barcelona, Ariel.

ROTHBARD, Murray (1995). An Austrian Perspective on the History of Economic Thought. Cheltenham, Edward Elgar Publishing.

RUBIN, Isaak Ilich (2007). Essays on Marx's Theory of Value. Calcuta, Harper CoIlins.

SIMMEL, Georg (2013). Filosofía del dinero. Madrid, Capitán Swing.

SMITH, Adam (1999). Investigación sobre la naturaleza y causas de la riqueza de las naciones. Barcelona, Planeta deAgostini.

STUART MILL, John (1997). Ensayos sobre algunas cuestiones disputadas en economía política. Madrid, Alianza Editorial.

VIRNO, Paolo (2003) Gramática de la multitud. Madrid, Traficantes de Sueños. 